\title{
A new effect of luminance gradient on achromatic simultaneous contrast
}

\author{
TIZIANO AGOSTINI and ALESSANDRA GALMONTE \\ University of Trieste, Trieste, Italy
}

\begin{abstract}
A new effect in the domain of achromatic simultaneous contrast has been observed. A middle gray region placed at the center of an area filled by a linear achromatic gradient from black (outer part) to white (inner part) is perceived as being much darker than an identical middle gray region surrounded by a reversed gradient. By using a matching task in two experiments, it has been shown that this phenomenon is much stronger than the classical achromatic simultaneous contrast effect. The new effect is interpreted in terms of the albedo hypothesis.
\end{abstract}

The different amounts of light coming from surfaces to the eyes can be produced either by changes in the level of illumination or by changes in the surfaces' color. This fact has led to the classification of edges into two categories: illumination edges and reflectance edges. At the retinal level, this distinction loses its meaning, since there are only luminance discontinuities. However, the information about the two types of edges is recovered by the visual system at the perceptual level. In fact, it has been shown that manipulating edge appearance affects, in general, the perception of surface color and, in particular, the size of achromatic simultaneous contrast. Achromatic simultaneous contrast can be defined as an increase in the perceived lightness difference between two physically identical regions surrounded by backgrounds having different intensities.

Burgh (1964) produced an increase of achromatic contrast by blurring the edge of a gray test patch in a classical simultaneous contrast configuration. The blur was obtained by throwing the projector out of focus in coincidence with the edge of the gray target region.

In his well-known corrugated Mondrian configuration, Adelson (1993) demonstrated that a simple modification of edge appearance greatly alters the strength of simultaneous contrast. This result was explained by assuming that the display is perceived as a three-dimensional (3-D) structure with different amounts of perceived illumination falling on the different planes.

Schirillo and Shevell (1997) measured a similar increase of contrast also. They found that the achromatic color of a test patch changes depending on whether a remote luminance edge is inferred to be the result of a local change in reflectance or the result of a more global change of illu-

This work was supported by MURST Grant MM11197484. We are extremely grateful to James Hoffman, Richard Bowen, and two anonymous reviewers for their helpful comments. Correspondence concerning this article should be addressed to T. Agostini, Dipartimento di Psicologia, Università di Trieste, via S. Anastasio, 12, 34134 Trieste, Italy (email: agostini@univ.trieste.it). mination. A test patch was matched with a lower luminance value in the no perceived illumination edge condition, as compared with that obtained in the perceived illumination edge condition.

All these works have shown that the size of achromatic contrast is altered largely by edge appearance. In this domain of studies, a new effect has been observed (presented for the first time at ARVO; Agostini \& Galmonte, 1997b). When two identical middle gray squares are placed at the center (respectively, white or black) of two checkerboardsthe first formed by white diagonal checks and the others filled by a linear achromatic gradient from black (outer part) to white (inner part), whereas the second checkerboard is the photographically reversed version of the first one-the gray target surrounded by white appears much darker than that surrounded by black (see Figures 1A and $\left.1 \mathrm{~B}^{1}\right)$. Even though local stimulation is identical, this phenomenon is much stronger than the classical achromatic simultaneous contrast effect, which is generally weak (for a review of the literature about the magnitude of the contrast effect, see Agostini \& Bruno, 1996).

Two experiments were performed. The first served to measure the size of this new effect, whereas the second served to determine the role of the gradient by removing the white and the black areas surrounding the tested region of Figures 1A and 1B.

\section{EXPERIMENT 1}

This experiment was performed to measure the size of the new effect.

\section{Method}

Observers. Sixty University of Trieste undergraduate or graduate students were tested, 15 in each of four conditions: Conditions 1 and 2 were conducted to measure the size of the new effect; Conditions 3 and 4 served as controls, as well as to establish a baseline, for Conditions 1 and 2. All the observers were volunteers, and all were naive as to the purpose of the experiment.

Apparatus and Stimuli. All the stimuli were displayed on a high-resolution CRT trinitron monitor $(1,280 \times 1,024$ pixels $)$ con- 
A

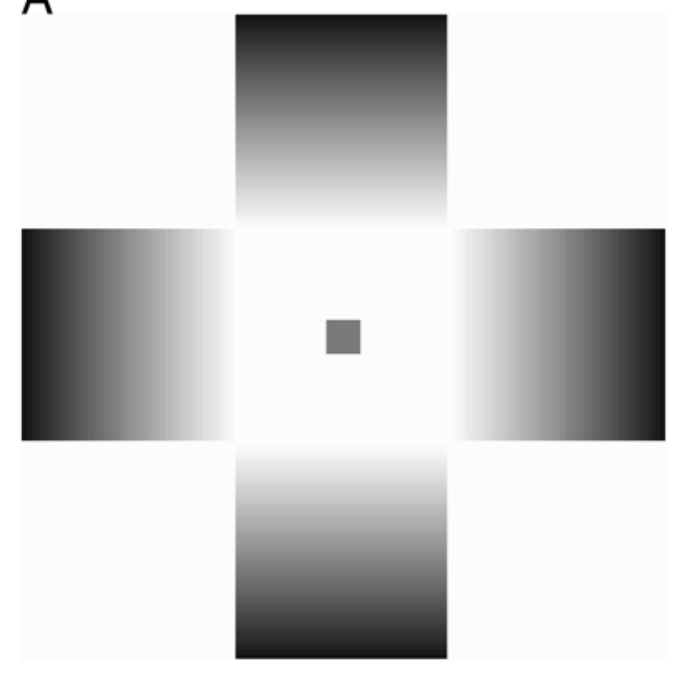

C

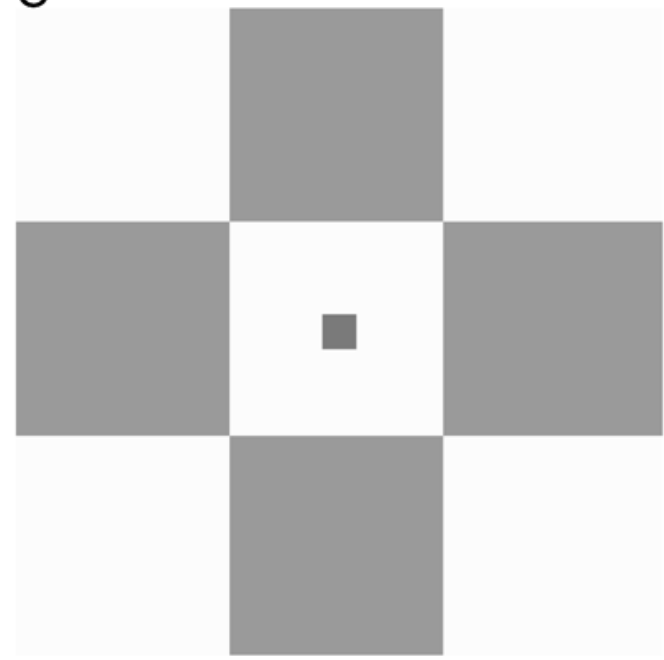

B
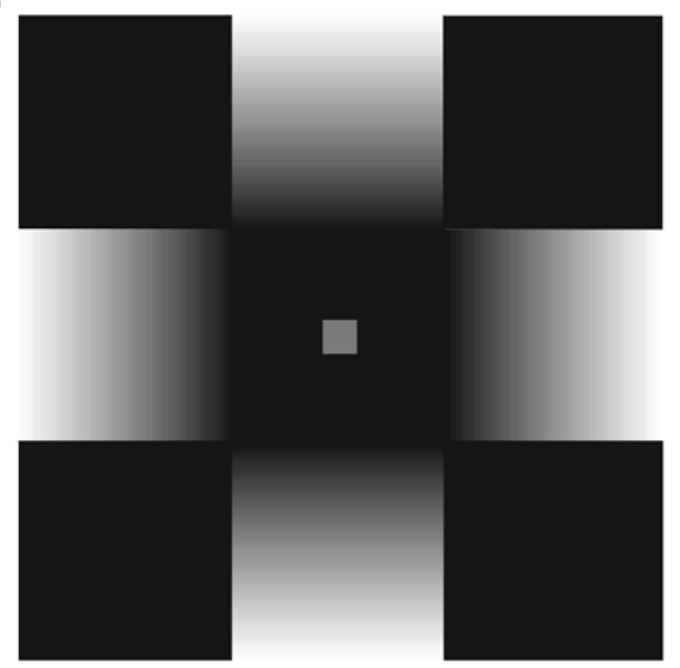

D

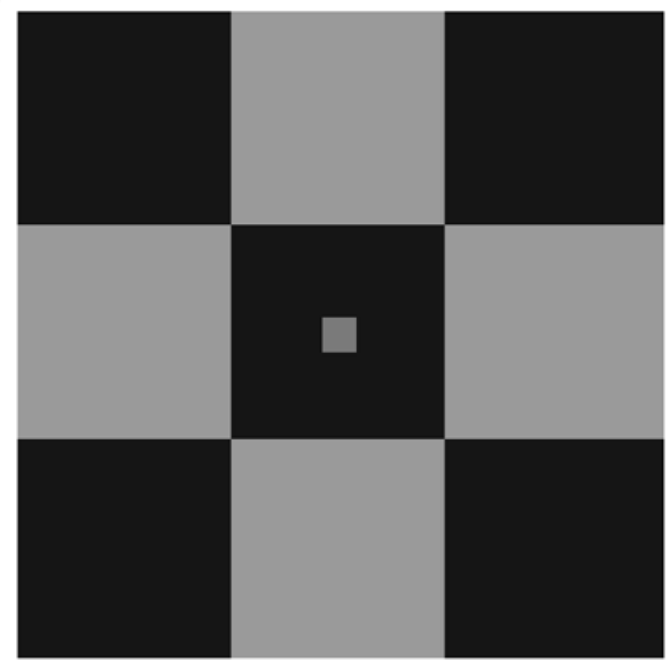

Figure 1. The four conditions tested in Experiment 1.

trolled by a Macintosh Performa 630. Luminance and chromaticity were controlled. In both experiments, the stimuli were presented on a background having a luminance of $66 \mathrm{~cd} / \mathrm{m}^{2}(9.5 \mathrm{~s} /$, that is, simulated Munsell units) and appeared in a $10^{\circ} \times 7.15^{\circ}$ visual angle window.

The experimental display was formed by 9 squares $\left(2.15^{\circ}\right.$ each) arranged in such a way as to form a squared checkerboard $\left(6.45^{\circ}\right)$. The target was a square $\left(0.28^{\circ}\right)$ placed at the center of the checkerboard. A vertical Munsell scale ${ }^{2}$ was simulated at $1.57^{\circ}$ from the outer border of the experimental display. The scale was formed by 17 squares $\left(0.28^{\circ}\right.$ each) ranging from 1.5 to $9.4 \mathrm{~s} /$ with a constant increment of $0.5 \mathrm{~s} /$, except for the last, which was a $0.4 \mathrm{~s} /$ increment. The distance between the outer borders of two adjacent squares was $0.11^{\circ}$. The scale was always presented on a white background.

Condition 1. As is depicted in Figure 1A, the five diagonal squares of the checkerboard were $9.4 \mathrm{~s} /$, the target was $5.5 \mathrm{~s} /$, and a linear gradient (inward progression) ranging from 1.5 to $9.4 \mathrm{~s} /$ filled the four remaining squares.
Condition 2. This condition was the reversed version of Condition 1 (Figure 1B). The five diagonal squares of the checkerboard were $1.5 \mathrm{~s} /$, the target was $5.5 \mathrm{~s} /$, and a linear gradient (inward progression) ranging from 9.4 to $1.5 \mathrm{~s} /$ filled the four remaining squares.

Condition 3. This condition was the control of Condition 1 (see Figure $1 \mathrm{C}$ ). The five diagonal squares of the checkerboard were $9.4 \mathrm{~s}$, the target was $5.5 \mathrm{~s} /$, and the four remaining squares were filled by the average luminance of the gradient $\left(27 \mathrm{~cd} / \mathrm{m}^{2} ; 6.6 \mathrm{~s} /\right)$.

Condition 4. This condition was the control of Condition 2 (see Figure 1D). The five diagonal squares of the checkerboard were $1.5 \mathrm{~s} /$, the target was $5.5 \mathrm{~s} /$, and the four remaining squares were filled by the average luminance of the gradient $\left(27 \mathrm{~cd} / \mathrm{m}^{2} ; 6.6 \mathrm{~s} /\right)$.

Procedure. The observers were tested individually. Viewing was binocular. The observers were seated $2 \mathrm{~m}$ away from the computer screen, in a dark experimental room. They were first asked to describe the experimental display; during this time, the simulated Munsell scale was not visible. Then they performed a lightness match for the central target by pointing to one of the patches on the 
scale. They were also allowed to choose an intermediate value between two adjacent patches on the simulated Munsell scale. The position of the scale was counterbalanced. Each observer performed one match for one condition only.

\section{Results and Discussion}

Figure 2 depicts the observers' mean matches for the central targets (simulated Munsell values). A factorial analysis of variance showed a highly significant difference among the four conditions $[F(3,56)=148.2, p<.0001]$. Fisher's post hoc comparisons were all significant ( $\alpha=.05$, with $p$ always lower than .005).

Even though local stimulation is identical, this striking effect is three times larger than that obtained in the comparison conditions ( 3.75 vs. $1.25 \mathrm{~s} /$ ), where the first value refers to the combined simultaneous contrast magnitude for Conditions 1 and 2 and the second value refers to the total effect of Conditions 3 and 4.

These results indicate that achromatic simultaneous contrast is greatly affected by the presence of a linear gradient. Furthermore, it must be noted that the scale was always surrounded by white, and for this reason we expected no contrast effect for all the experimental displays in which the gray target was surrounded by white. Sur- prisingly, when the central white area was influenced by the luminance gradient, the gray patch was perceived as almost 1 Munsell unit darker than its objective value.

\section{EXPERIMENT 2}

This experiment was conducted to verify whether the new effect also takes place when the target is surrounded only by a linear square-shaped gradient.

\section{Method}

Observers. Thirty University of Trieste undergraduate or graduate students were tested, 15 in each of two conditions. All the observers were volunteers, and all were naive as to the purpose of the experiment. None of them had participated in the previous experiment.

Apparatus and Stimuli. The apparatus was the same as in the previous experiment. A large square background $\left(6.45^{\circ}\right)$ with a target $\left(0.28^{\circ}\right)$ in the middle formed the experimental display. All the parameters used for the simulation of the Munsell scale were the same as those in the previous experiment.

Condition 1. As is depicted in Figure 3A, the target was $5.5 \mathrm{~s} /$, and the large square was filled by a linear square-shaped gradient (inward progression) ranging from 1.5 to $9.4 \mathrm{~s} /$.

Condition 2. This condition was the reversed version of Condition 1 (see Figure 3B). The target was $5.5 \mathrm{~s} /$, and a linear gradient (inward progression) ranging from 9.4 to $1.5 \mathrm{~s} /$ filled the large square.

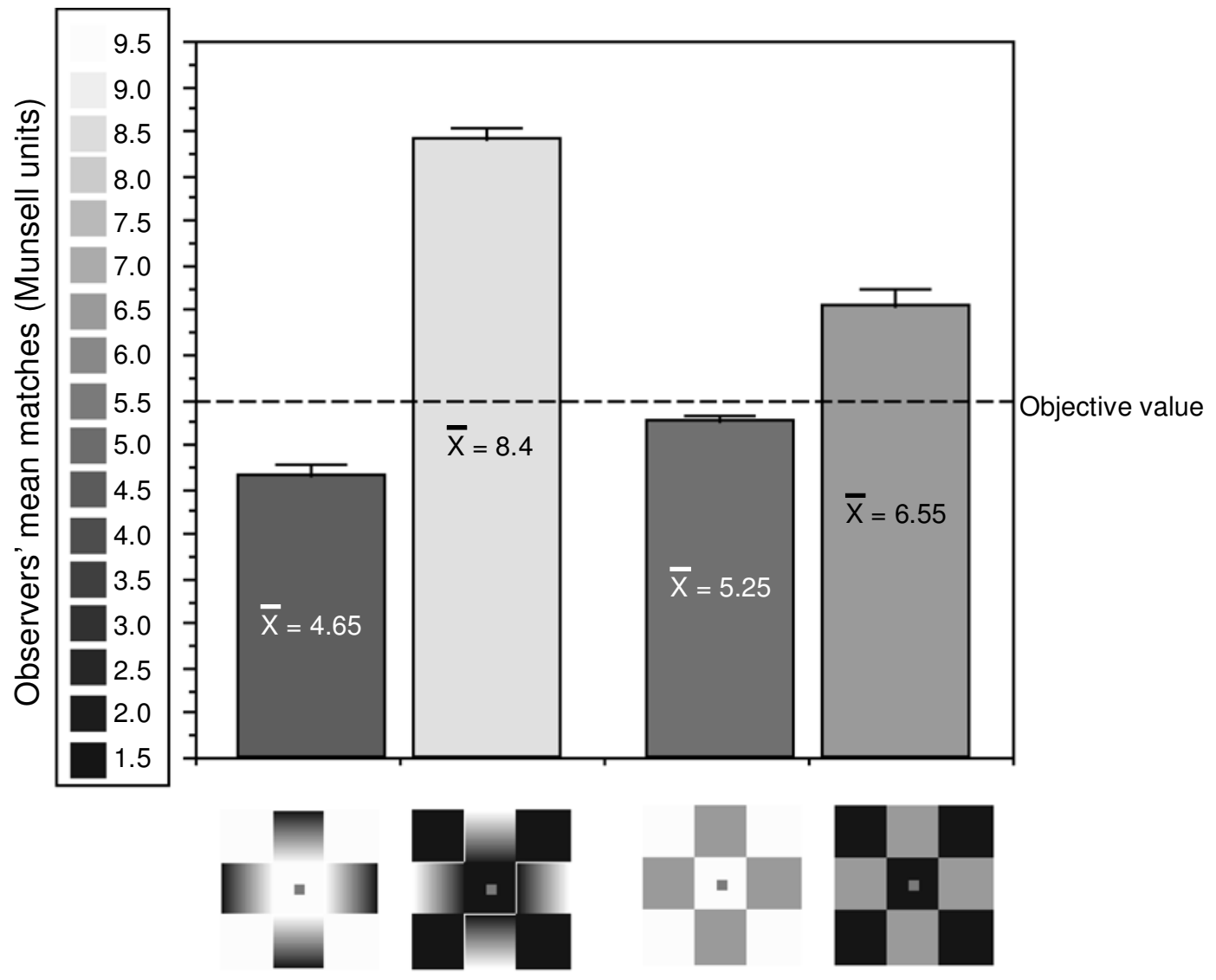

Figure 2. The results obtained in Experiment 1. The heights of the bars indicate the observers' mean matches in Munsell units for each condition (the values are reported inside the bars). The dashed line represents the objective Munsell value of the target region. An error bar indicates the standard error of the mean. 

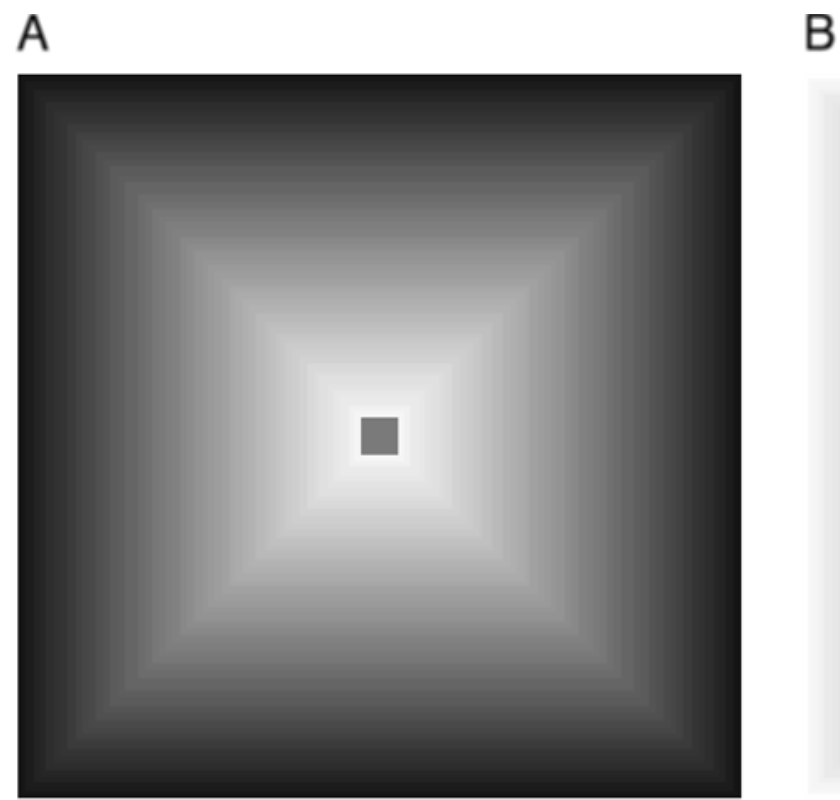

Figure 3. The two conditions tested in Experiment 2.

In this experiment, there were neither control nor baseline conditions, since by replacing the gradients with their weighted average luminance, the direction of the induction effects should be reversed.

Procedure. The procedure was the same as that in the previous experiment.

\section{Results and Discussion}

Figure 4 depicts the observers' mean matches for the central targets (simulated Munsell values). An unpaired $t$ test showed a highly significant difference between the two conditions $[t(28)=21.4, p<.0001]$.

The results of this experiment show that the achromatic simultaneous contrast is also greatly affected when the target is directly surrounded by a linear square-shaped gradient.

The size of the effect is identical in Experiments 1 and 2 (no significant difference was found in an unpaired $t$ test performed on the differences between the observed matches of Conditions 1 and Conditions 2), although in Experiment 2 it might have been smaller. This result was to be expected, since the average luminance of the region surrounding the target in Condition 1 of Experiment 1 is higher than that in Condition 1 of Experiment 2, and the average luminance of the region surrounding the target in Condition 2 of Experiment 1 is lower than that in Condition 2 of Experiment 2. Also in this case, in the condition in which the intensity of the luminance gradient increases toward the center, the gray patch was perceived as almost 1 Munsell unit darker than its objective value, even though the matching scale was surrounded by white.

\section{GENERAL DISCUSSION}

The contrast effect reported in this paper is the strongest that has been observed. Its size is three times larger than that obtained in the baseline conditions (3.75 vs. $1.25 \mathrm{~s} /$ ).
When the white and the black regions are removed, the size of the effect does not change. These results cannot be interpreted within the framework of lateral inhibition theories. According to these theories, the lightness of a region is the result of a process of inhibition determined only by the intensity of its surround (see Agostini \& Proffitt, 1993). Since we found an enormous difference between the experimental and the control conditions, lateral inhibition accounts cannot be applied to our results.

Displays 1 and 2 of Experiment 1 show a particularly evident effect of bright glare and dark mist, respectively (Kennedy, 1976; Zavagno, 1999). However, these factors are not necessary for the contrast effect to arise. In fact, in the displays used in Experiment 2, phenomenal glare and dark mist, if any, are really weak. Since the results of Experiment 2 do not statistically differ from those obtained in Experiment 1, it is possible to conclude that these factors are not crucial.

Gilchrist et al.'s (1999) theory explains lightness contrast by applying the highest luminance rule, which states that the highest luminance in a scene is perceived as white. According to this rule, lightness contrast occurs because the gray patch surrounded by black is the highest luminance in that framework and, for this reason, the visual system tends to assign to the gray region the value of "white." This means that almost all the contrast effect is shown by the gray surrounded by black. The model also predicts a small contrast effect on the gray patch surrounded by white, owing to a scale normalization component. Luminance gradients exert a segregative role in belongingnessterms, and therefore, the illusionis strengthened. However, the model, which, in its current form, makes no reference to the role of perceived illumination, does not say why luminance gradients should be a segregation factor. 


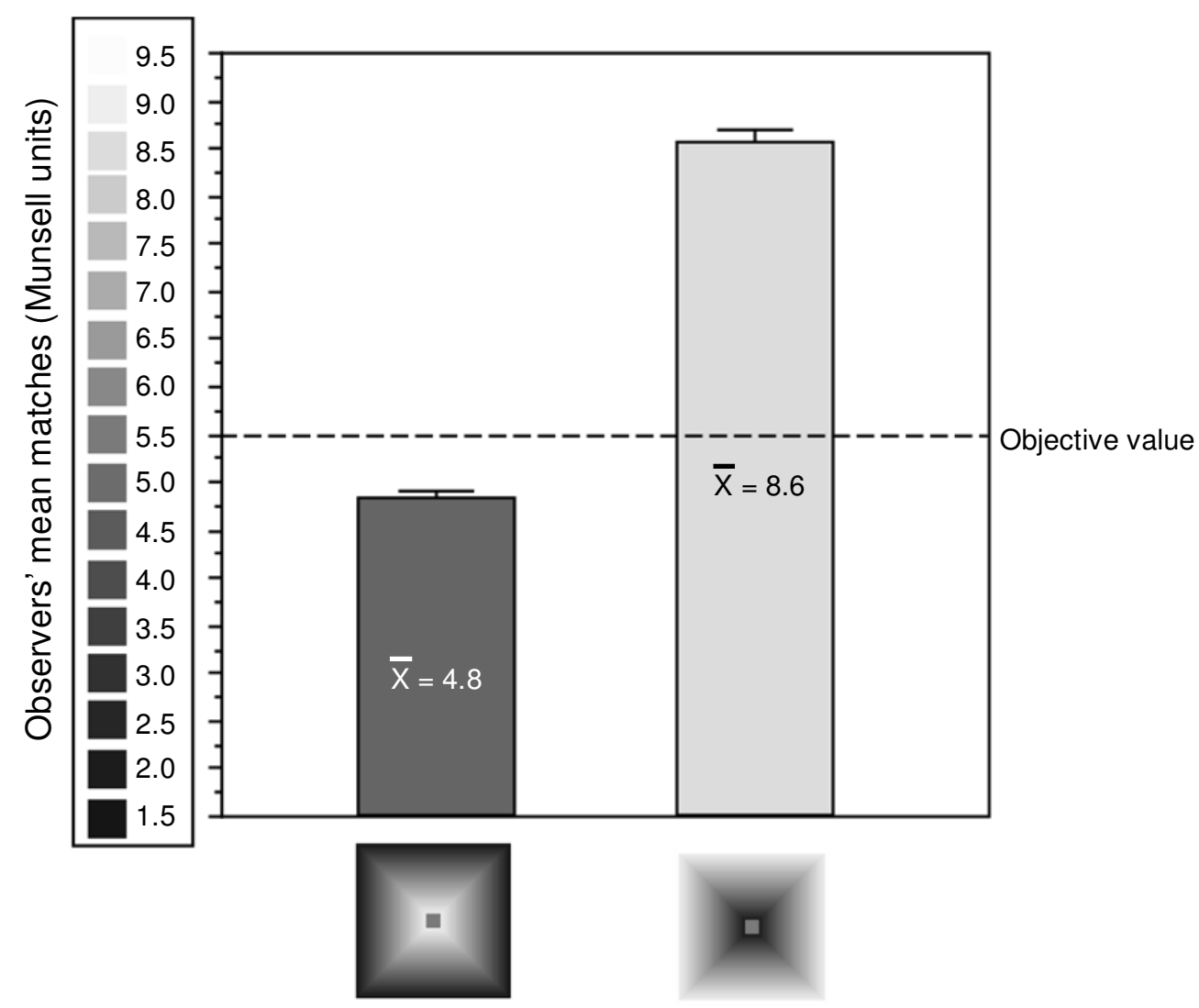

Figure 4. The results obtained in Experiment 2. The heights of the bars indicate the observers' mean matches in Munsell units for each condition (the values are reported inside the bars). The dashed line represents the objective Munsell value of the target region. An error bar indicates the standard error of the mean.

Another possible way to explain the effect reported in this paper is to assume that the luminance gradient is perceived as a change in the illumination level, rather than as a change in the surfaces' reflectance.

This interpretation is supported by the descriptions given by the observers. For instance, in Experiment 1, Display $1 \mathrm{~A}$ was spontaneously described by 10 out of $15 \mathrm{ob}-$ servers as a black cross or a checkerboard illuminated from its center and shadowing off to its periphery, whereas Display 1B was spontaneously described by 13 out of 15 observers as a white cross or a checkerboard toning down from its periphery to its center, which is in deep shade. In Experiment 2, all the observers described the display depicted in Figure 3A as a black pyramid illuminated from its center. In Figure 3B, all the observers described the display as a white tunnel in deep shade in its farthest part. In both experiments, before performing the match, all those observers who described the experimental display in terms of illumination spontaneously reported that they perceived the target at the center of the bright region as black or very dark gray and the one at the center of the dark region as white or very light gray.

It should be noted that the observers always gave a 3-D description of the displays of Experiment 2, whereas this never occurred when they described the displays of Experiment 1. Further experiments are needed to control for this factor.

Given these considerations, it appears that the visual system classifies the luminance gradient in our displays as if it is an illumination gradient. In Displays $1 \mathrm{~A}$ and $3 \mathrm{~A}$, the luminance of the gray target is a decrement in comparison with its bordering luminance, but since the context in which it is perceived appears as a black region brightly illuminated, the target, to be seen within the same apparent illumination, must be perceived as darker than it really is. In Figures $1 \mathrm{~B}$ and 3B, instead, the gray target is an increment relative to its surrounding luminance, but since its context is perceived as a white area in deep shade, the target, to be seen within the same apparent illumination must be perceived as lighter than it really is. This interpretation is congruent with the logic of the albedo hypothesis (Beck, 1972; Kozaki, 1965; Kozaki \& Noguchi, 1976; Logvinenko \& Menshikova, 1994; Noguchi \& Kozaki, 1985; Oyama, 1968), which states that there is an invariant relationship between perceived surface lightness and perceived illumination level. An error in perceived lightness goes with an equal but opposite error in perceived illumination. 
Further observations showed that, given a linear function of the gradient, the shape of the gradient and that of the display do not affect the size of the effect, for instance, when a circle-shaped gradient is introduced in a classical disk/annulus configuration(Agostini \& Galmonte, 1997a).

\section{REFERENCES}

Adelson, E. H. (1993). Perceptual organization and the judgement of brightness. Science, 262, 2042-2044.

Agostini, T., \& Bruno, N. (1996). Lightness contrast in CRT and paper-and-illuminant displays. Perception \& Psychophysics, 58, 250258.

Agostini, T., \& Galmonte, A. (1997a). Luminance gradients, perceived illumination, and lightness perception. Review of Psychology, 4, 3-6.

Agostini, T., \& Galmonte, A. (1997b). A new effect of gradient on lightness [Abstract]. Investigative Ophthalmology \& Visual Science, 38, S895.

Agostini, T., \& Proffitt, D. R. (1993). Perceptual organization evokes simultaneous lightness contrast. Perception, 22, 263-272.

BEcK, J. (1972). Surface color perception. Ithaca, NY: Cornell University Press.

BurGH, P. (1964). Peripheral viewing and simultaneous contrast. Quarterly Journal of Experimental Psychology, 16, 257-263.

Gilchrist, A. L., Kossy fidis, C., Bonato, F., Agostini, T., Cataliotti, J., Li, X., Spehar, B., Annan, V., \& Economou, E. (1999). An anchoring theory of lightness perception. Psychological Review, 106, 795-834.

Kennedy, J. M. (1976). Sun figure: An illusory diffusive contour resulting from an arrangement of dots. Perception, 5, 479-481.
KozaKi, A. (1965). The effect of co-existent stimuli other than the test stimulus on brightness constancy. Japanese Psychological Research, 7, 138-147.

Kozaki, A. \& Noguchi, K. (1976). The relationship between perceived surface-lightness and perceived illumination. Psychological Research, 39, 1-16.

Logvinenko, A., \& Menshikova, G. (1994). Trade-off between achromatic colour and perceived illumination as revealed by the use of pseudoscopic inversion of apparent depth. Perception, 23, 1007-1024.

NogUchi, K., \& KoZAKI, A. (1985). Perceptual scission of surfacelightness and illumination: An examination of the Gelb effect. Psychological Research, 47, 19-25.

Oyama, T. (1968). Stimulus determinants of brightness constancy and the perception of illumination. Japanese Psychological Research, 10, 146-155.

Schirillo, J. A., \& Shevell, S. K. (1997). An account of brightness in complex scenes based on inferred illumination. Perception, 26, 507518.

ZaVagno, D. (1999). Some new luminance-gradient effects. Perception, 28, 835-838.

\section{NOTES}

1. In all the figures, some level of gray could have been lost in the printing process.

2. The Munsell scale follows a logarithmic function.

(Manuscript received December 17, 1998; revision accepted for publication July 2, 2001.) 